\title{
Metastatic epithelioid hemangioendothelioma to the bone marrow with associated myelofibrosis resembling primary myelofibrosis
}

Venkata Rakesh Sethapati ${ }^{1}$, Wei Cui ${ }^{1}$ and Da Zhang $^{1, *}$

' Department of Pathology and Laboratory Medicine, The University of Kansas Medical Center, 3901 Rainbow Blvd., MS 3045, Kansas City, KS 66160, USA

\begin{abstract}
Epithelioid hemangioendothelioma (EHE) is a low-grade malignant vascular endothelial tumor. EHE of the spleen is rare and most often represents metastasis of EHE from other sites, or disseminated disease or multifocal disease. We report a case of EHE presenting as a splenic mass with multiple foci of bone lesion and mimicking primary myelofibrosis in the bone marrow biopsy.
\end{abstract}

Keywords: epithelioid hemangioendothelioma; spleen; myelofibrosis

\section{Introduction}

Epithelioid hemangioendothelioma (EHE) is a rare low-grade malignant tumor nosologically classified as intermediate between highly malignant angiosarcoma and benign hemangioma [1-3]. Subsequent studies revealed that EHE may originate in many parenchymal organs, and may be either solitary and unifocal or multifocal involving more than one organ $[1,4]$. The spleen seems to be rarely involved and is most often a manifestation of a multisystemic dissemination from a defined primary site or a multifocal, multiorgan disease [1].

Here we report a case of splenic EHE with clinical findings of splenomegaly, thrombocytopenia, anemia and extramedullary hematopoiesis and initial bone marrow biopsy was suggesting primary myelofibrosis. Subsequent imaging studies revealed multiple foci of bone lytic lesions. Repeat bone marrow biopsy showed focal prominent atypical vascular proliferation, consistent with EHE involving the bone marrow. This case was a diagnostic challenge, illustrating the overlapping features between MF and myelofibrosis secondary to EHE.

\section{Case report}

A 40-year-old woman initially presented with cough, respiratory infection and low-grade fever. Her peripheral blood smear showed normocytic anemia, anisopoikilocytosis, erythroblastosis with nucleated red blood cells, absolute neutrophilia with left shift and thrombocytopenia and the CBC data showed Hgb $8.7 \mathrm{~g} / \mathrm{dL}$; MCV 94.6 FL; RDW 25.3\%; WBC $19.9 \times 10^{9} / \mathrm{L} ; \mathrm{PLT} 26 \times 10^{9} / \mathrm{L}$. A bone marrow biopsy was performed (Figure 1) showing a hypercellular marrow (100\%) with marked fibrosis and trilineage hematopoiesis with increase hyperlobated megakaryocytes, and 1\% blasts. Reticulin and trichrome stain showed markedly increased reticulin fibrosis with collagen deposit that led to the diagnosis of bone marrow fibrosis (WHO grade MF 3/3). The bone marrow flow cytometry revealed negative immunophenotypic study. Overall this was concerning for primary myelofibrosis. Imaging studies showed a massively enlarged spleen suspicious for extramedullary hematopoiesis. There were no enlarged lymph nodes. Consequential uncontrolled abdominal pain led to a splenectomy.

The surgically resected spleen weighed 1866 grams and measured $27.2 \times 17.3 \times 5.8 \mathrm{~cm}$. There were three yellowtan nodules, ranging in greatest dimension from 0.6 to $0.2 \mathrm{~cm}$. One nodule was located along the periphery and the two remaining nodules were located more centrally. Microscopically, the specimen demonstrated an infiltrative process, comprised of mildly atypical spindle cells that had elongated nuclei and well-developed eosinophilic

*Corresponding author: Da Zhang, Department of Pathology and Laboratory Medicine, The University of Kansas Medical Center, 3901 Rainbow Blvd., MS 3045, Kansas City, KS 66160, USA. Tel.: 913-588-0388; Fax: 913-588-8780; Email: dzhang@kumc.edu

Received 17 August 182018 Revised 11 October 2018 Accepted 20 October 2018 Published 30 October 2018

Citation: Sethapati VR, Cui W, Zhang D. Metastatic epithelioid hemangioendothelioma to the bone marrow with associated myelofibrosis resembling primary myelofibrosis. J Mod Hum Pathol. 2018; 3(6):18-21. DOI: 10.14312/2397-6845.2018-6

Copyright: (c) 2018 Sethapati VR, et al. Published by NobleResearch Publishers. This is an open-access article distributed under the terms of the Creative Commons Attribution License, which permits unrestricted use, distribution and reproduction in any medium, provided the original author and source are credited. 
cytoplasm. The tumor cells were arranged in an anastomosing pattern along with solid aggregates of oval and occasional polygonal cells, forming only occasional vascular slit spaces that contained erythrocytes. On higher power these cells had intracytoplasmic lumina and occasionally contained internalized erythrocytes. Despite this mild atypia there was no significant mitotic activity. Much of the red pulp was replaced by this infiltrate and also contained occasional foci of residual white pulp. Extensive extramedullary hematopoiesis was present. The spindle cells infiltrating the spleen were immunohistochemically positive for CD31, CD34, CD8 and Cyclin D1. SMA stained stromal components but also stained some of the tumor cells. CD68 and CD163 reacted with the background histocyte and residual littoral cells. The tumor cells were negative for CD20, CD3, HHV8, EBV ISH, CD21, CD35, CD1a, S100, MCT, and ALK. Due to low-grade morphology of vascular tumor, a diagnosis of hemangioma was rendered by the consulted institution. The nature of extensive extramedullary hematopoiesis was not clear since it could be present in vascular tumor in addition to myeloproliferative neoplasms. Primary myelofibrosis or other myeloproliferative neoplasms were still considered as the most likely diagnosis, best correlating with her extramedullary hematopoiesis and bone marrow findings. However, molecular studies for JAK2, calreticulin, BCR-ABL, MPL and PDGFR mutations were all negative. Cytogenetic studies showed a normal karyotype. Hematologic neoplasms targeted gene panel which contained 141 genes by NGS showed zero pathogenic variants and two variants of uncertain significance (DNMT3A and KMT2C).

The patient temporarily did better but she continued to have persistent anemia and thrombocytopenia that warranted regular transfusions. A second bone marrow biopsy had similar results as the first. The patient's history was pertinent for rheumatoid arthritis and labs were positive for ANA and antihistone antibody. A second opinion was consulted at a different institution and She was told her hematological findings were possibly due to an underlying mixed connective tissue disease causing an autoimmune cytopenia. She was started on steroids but her platelets remained below $20 \times 10^{9} /$. Her declining condition necessitated a third bone marrow biopsy.

Microscopic examination of the third core biopsy showed a hypercellular marrow partially replaced by a vascular neoplasm composed of spindled and epithelioid cells forming slit-like vascular channels and capillaries with increased hyperlobated megakaryocytes and occasional separated lobes.

A reticulin stain revealed a marked increase in fibrosis in areas involved by the vascular neoplasm; the uninvolved bone marrow showed no fibrosis. A trichrome stain demonstrated collagen in the fibrotic areas associated with the vascular neoplasm. The spindle cells forming the vascular neoplasm mimicked fibroblasts proliferation in a background of real secondary fibroblast proliferation. These spindle cells were positive for CD31, CD34, factor VIII, and FLI-1. The immunophenotype and morphology of the bone neoplasm were histologically similar to the lesion in the spleen; a diagnosis of EHE was finally rendered.
Further imaging showed extensive lytic lesions in hip bones, bilateral extremities and throughout the calvarium. The patient was treated with paclitaxel and radiation therapy but extensive bone involvement by EHE led to refractory anemia and thrombocytopenia. Unfortunately, she was not responding to the treatment and passed away six months later.

\section{Discussion}

The present case presented as a diagnostic challenge. Initially, the persistent anemia and thrombocytopenia were concerning for primary hematological malignancy. Hypercellular marrow with increased and atypical megakaryocytes, extensive fibrosis and corresponding peripheral smear findings resembled primary myelofibrosis. Molecular studies for JAK2, calreticulin, BCR-ABL, MPL and PDGFR mutations were all negative. Cytogenetic studies showed a normal karyotype. Hematologic neoplasms targeted gene panel which contained 141 genes by NGS showed zero pathogenic variants and two variants of uncertain significance (DNMT3A and KMT2C). DNMT3A is often mutated in myeloid disease; however, our case is not the typical Arg882 variant. KMT2C as part of the MLL signaling pathway is also implicated in myeloid disease, but the particular variant in this patient is not known to any myeloid disease.

The patient's splenomegaly was considered to be yet another cause of the hematologic problems and accordingly a splenectomy was performed. The process of making a right diagnosis of EHE was rather challenging until imaging studies and a repeat bone marrow biopsy demonstrated metastatic disease. The tumor cells were positive for CD31, CD34 and FLI-1 and molecular study for myeloproliferative neoplasm was negative, thus, these findings enabled us to make the correct diagnosis of multifocal splenic EHE with osseous metastasis.

Splenic EHE may occur in two forms: an isolated form of disease or as a manifestation of a multifocal and disseminated end-stage form of the disease [5]. Splenic EHE as part of disease dissemination or multifocal disease is much more common than solitary EHE. The literature contains only a handful of case reports of isolated splenic EHE in which the tumor was limited to the spleen (Table 1). One was a nine-year-old pediatric patient with a single tumor in the spleen [6], and the other was a 24-year-old woman with a multicentric tumor in the spleen [7]. Both cases had no complications and had a complete recovery after splenectomy with no evidence of metastasis.

In our patient, splenic EHE was associated with multifocal bone metastasis. A similar case with splenic presentation was reported by Emamaullee et al. [8] who described a resected EHE of the spleen which led to the subsequent diagnosis of a liver EHE, hepatectomy, and liver transplantation. In contrast to that case, which had a reported favorable outcome, the multiplicity of bone metastasis made surgical resection in our patient impossible.

Utilizing immunohistochemical stains such as CD31, CD34 and FLI-1 will be helpful in distinguishing EHE from other metastatic malignancies [9]. 

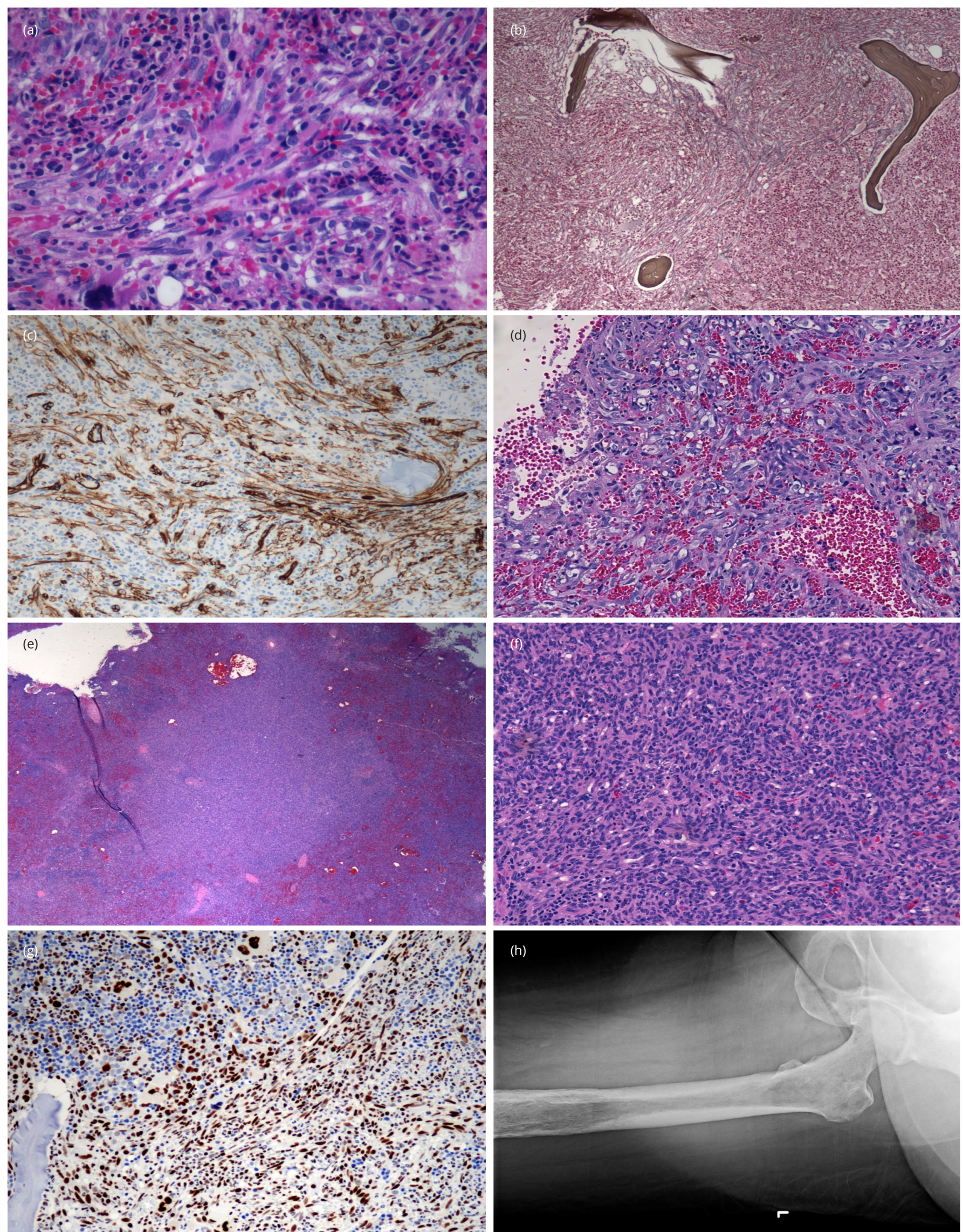

Figure 1 (a) The first bone marrow biopsy showed spindle cell proliferation with scattered hematopoietic elements; (b) Reticulin stain from the first bone marrow biopsy showed increase reticulin stain and surrounded the individual spindle cells; (c) CD34 stain highlighted the scattered blasts and vascular proliferation; (d) The third bone marrow biopsy showed prominent vascular proliferation with spindle cells and red blood cells in the space; (e) Splenectomy specimen showed a nodular mass surrounded by normal splenic tissue; $(f)$ Spleen mass at high power $(40 \mathrm{x})$ showed spindle cell proliferation with scattered vascular space; (g) FLI-1 immunohistochemical stain shows nuclear staining pattern in the tumor cells; (h) X-ray of left femur shows numerous diffuse scattered lytic lesions. 
Table 1 Summary of reported spleen hemangioendothelioma cases.

\begin{tabular}{|c|c|c|c|c|c|c|}
\hline Case & Gender/ age & Splenic pathology & Metastasis & Treatment & Outcome & References \\
\hline 1 & $\mathrm{M} / 9$ & Epithelioid and spindle cell HE & $\begin{array}{l}\text { Liver } \\
\text { (unknown primary) }\end{array}$ & $\begin{array}{l}\text { Splenectomy with } \\
\text { liver biopsy }\end{array}$ & $\begin{array}{l}\text { Mortality due } \\
\text { to consumptive } \\
\text { coagulopathy }\end{array}$ & [9] \\
\hline 2 & $F / 9$ & HE (multicentric) & None & Partial splenectomy & $\begin{array}{l}\text { No recurrence in } 15 \\
\text { months }\end{array}$ & {$[6]$} \\
\hline 3 & $F / 28$ & Splenic littoral cell HE (multicentric) & Liver & Splenectomy & $\mathrm{N} / \mathrm{A}$ & {$[12]$} \\
\hline 4 & $\mathrm{~F} / 67$ & Composite HE (multicentric) & Liver & $\begin{array}{l}\text { Splenectomy and } \\
\text { chemotherapy }\end{array}$ & $\mathrm{N} / \mathrm{A}$ & {$[11]$} \\
\hline 5 & $F / 24$ & Spindle cell HE (multicentric) & None & Splenectomy & $\begin{array}{l}\text { No recurrence in one } \\
\text { year }\end{array}$ & {$[7]$} \\
\hline 6 & $\mathrm{~F} / 40$ & Epithelioid HE (mutlicentric) & Bone & Splenectomy & Deceased & Our case \\
\hline
\end{tabular}

Abbreviation: $\mathrm{HE}$ : hemangioendothelioma.

The main treatment for EHE is surgical resection. If the tumor is small, confined and limited in number, surgical resection usual has good prognosis. The treatment modalities to metastatic EHE include cytotoxic and antiangiogenic chemotherapy regimens. The use of antiangiogenic medication is beneficial such as Bevacizumab and Apatinib. Due to the rarity of this disease, no standard therapy has been established.

\section{Conclusion}

EHE involving bone marrow is a diagnostic challenge. Not only can EHE induce secondary myelofibrosis around the tumor, but the individual spindle tumor cells are also characteristically surrounded by reticulin stain mimicking fibrosis. These findings along with reactive atypical megakaryocytes could potentially lead to an impression of primary myelofibrosis. The CD34 stain which is commonly used in dry tap aspirates will highlight the blasts, capillary endothelial cells as well as the spindle tumor cells of EHE. Additional immunohistochemical stains such as other vascular markers like FLI-1, CD31, factor VIII, imaging and molecular studies should be used to rule out a myeloproliferative neoplasm and thus distinguish metastatic EHE in the bone from primary myelofibrosis.

\section{Conflict of interest}

Authors declare no conflicts of interest related to this case report.

\section{References}

[1] Flucke U, Vogels RJ, de Saint Aubain Somerhausen N, Creytens DH, Riedl RG, et al. Epithelioid Hemangioendothelioma: Clinicopathologic, immunhistochemical, and molecular genetic analysis of 39 cases. Diagn Pathol. 2014; 9:131.

[2] Sardaro A, Bardoscia L, Petruzzelli MF, Portaluri M. Epithelioid hemangioendothelioma: An overview and update on a rare vascular tumor. Oncol Rev. 2014; 8(2):259.

[3] Weiss SW, Enzinger FM. Epithelioid hemangioendothelioma a vascular tumor often mistaken for a carcinoma. Cancer. 1982; 50(5):970-981.

[4] Studer LL, Selby DM. Hepatic Epithelioid Hemangioendothelioma. Arch Pathol Lab Med. 2018; 142(2):263-267.

[5] Tan Y, Yang X, Dong C, Xiao Z, Zhang H, et al. Diffuse hepatic epithelioid Hemangioendothelioma with multiple splenic metastasis and delayed multifocal bone metastasis after liver transplantation on FDG PET/CT images: A case report. Medicine (Baltimore). 2018; 97(22):e10728.
[6] Wang Z, Zhang L, Zhang B, Mu D, Cui K, et al. Hemangioendothelioma arising from the spleen: A case report and literature review. Oncol Lett. 2015; 9(1):209-212.

[7] Hazinedaroglu SM, Kayaoglu HA, Dulger F, Serinsoz E, Kuzu I, et al. Multicentric spindle cell hemangioendothelioma of spleen: Case report. Turk J Cancer. 2008; 38(1):30-33.

[8] Emamaullee JA, Nowak K, Beach M, Bacani J, Shapiro AMJ. Hepatic Epithelioid hemangioendothelioma presenting as an enlarging vascular lesion within the spleen. Case Rep Transplant. 2018; 2018:3948784.

[9] Gill R, O'Donnell RJ, Horvai A. Utility of immunohistochemistry for endothelial markers in distinguishing epithelioid hemangioendothelioma from carcinoma metastatic to bone. Arch Pathol Lab Med. 2009; 133(6):967-972.

[10] Goyal A, Babu SN, Kim V, Katariya S, Rao KL. Hemangioendothelioma of liver and spleen: trauma-induced consumptive coagulopathy.J Pediatr Surg. 2002; 37(10):E29.

[11] Yoda Y, Masaki O. A case of composite hemangioendothelioma arising from the spleen. Jpn J Clin Oncol. 2012; 42(8):770.

[12] He P, Yan XD, Wang JR, Guo RC, Zhang HB. Splenic littoral cell hemangioendothelioma: Report of a case with hepatic metastases and review of the literature. J Clin Ultrasound. 2014; 42(5):308-312. 